\title{
Reproductive performance in rats with different histories of malnutrition
}

\author{
BY JANINA R. GALLER \\ Department of Psychiatry, Boston University Medical Center, Boston, \\ Massachusetts 02118 USA \\ AND GARY ZARTARIAN \\ Department of Nutrition and Food Science, Massachusetts Institute of Technology, \\ Cambridge, Massachusetts 02139 USA
}

(Received 17 January 1980 - Accepted 19 September 1980)

\begin{abstract}
1. Reproductive competence, as measured by weight gain during pregnancy, duration of pregnancy and foetal outcome, was assessed in primiparous rats (4-6 months of age) with various histories of malnutrition.

2. Although weight gain during pregnancy was reduced in rats with intergenerational malnutrition, the duration of pregnancy and number of live births did not differ from well-fed controls, suggesting adaptation of these measures to long-term dietary deprivation.

3. Rats malnourished from birth through pregnancy (one generation of malnutrition) had fewer live births than did rats with chronic, intergenerational malnutrition or well-fed control rats.

4. Gestation was prolonged and weight gain accelerated among female rats with histories of intergenerational malnutrition, who had been provided with an adequate diet from birth through pregnancy.
\end{abstract}

Reproductive performance is compromised in rats with severe malnutrition. However, the effects of malnutrition of longer duration, including intergenerational malnutrition, on performance (as measured by duration of and weight gain during pregnancy, and foetal outcome) are not known. Studies have shown that women of short stature, presumably with life-long histories of inadequate nutrition, had higher risk of a compromised pregnancy than women of normal stature (Baird, 1947; Bernard, 1952). Compromised pregnancies include those with complications, perinatal mortality, and premature births (Baird \& Illsley, 1953). These findings suggest that nutritional history before pregnancy significantly affects foetal outcome.

Accordingly, we compared weight gain during pregnancy in rats with different histories of nutritional intake, including intergenerational malnutrition with or without dietary rehabilitation after birth, postnatal malnutrition, and adequate nutrition. Our results show that reproductive performance is most impaired by postnatal malnutrition, and that intergenerationally malnourished female rats adapt to achieve better reproductive performance.

\section{MATERIALS AND METHODS}

\section{Animals}

Hooded female rats from a colony originated by Stewart (1972) and Stewart et al. (1973) were tested for reproductive capacity after various nutritional treatments: (1) animals with intergenerational malnutrition ( $F_{n} / L P$; born to mothers with eighteen to twenty generations of dietary deficiency) were provided with a $75 \mathrm{~g}$ casein $/ \mathrm{kg}$ diet after weaning and were malnourished at the time of testing ( $n$ 15); (2) animals with postnatal malnutrition $\left(F_{1} / L P\right.$; 
born to well-nourished mothers and cross-fostered to malnourished mothers at birth) were also provided with a $75 \mathrm{~g}$ casein $/ \mathrm{kg}$ diet after weaning and had been malnourished for 5 months at the time of testing $(n 5)$; (3) rehabilitated animals $\left(\mathbf{R}_{1} / \mathrm{LP}\right.$; born to mothers with intergenerational malnutrition $\left(\mathrm{F}_{17-19}\right)$ and cross-fostered at birth to well-nourished mothers) were provided with a $250 \mathrm{~g}$ casein/kg diet after weaning $(n 10)$; (4) well-nourished control animals $\left(F_{n} / C\right.$; born to mothers who had received an adequate $(250 \mathrm{~g}$ protein $/ \mathrm{kg})$ diet $)$ were provided with a $250 \mathrm{~g}$ casein $/ \mathrm{kg}$ diet after weaning $(n 14)$.

\section{Housing}

Animals were housed in wire mesh cages each containing two to three rats in a room maintained at $22 \pm 2^{\circ}$, with $40-60 \%$ relative humidity (Belfort temperature-humidity gauge). The room had a reverse 12-h dark/12-h light cycle. The light period was from 20.00 hours to 08,00 hours, so that behavioural tests were done during the rats' waking period. Animals received 75 or $250 \mathrm{~g}$ casein $/ \mathrm{kg}$ diets ad lib. (Galler et al. 1979). Water contained $1 \mathrm{mg}$ tetracycline/ml as prophylaxis for respiratory disease.

\section{Breeding}

The $F_{n} / L P$ and $F_{1} / L P$ females were matched by initial weight, as were the $R_{1} / L P$ and $F_{n} / C$ females. Since $R_{1} / L P$ adult females are heavier than their controls of the same age, younger animals were used. Thus the mean ( $\pm \mathrm{SE}$ ) ages (months) of the various groups of females were: $F_{n} / L P 6 \cdot 2 \pm 1 \cdot 8, F_{n} / C 7 \cdot 1 \pm 1 \cdot 6, F_{1} / L P 7 \cdot 4 \pm 1 \cdot 0$ and $R_{1} / L P 4 \cdot 8 \pm 1 \cdot 8$. Half the subjects were mated over one estrous cycle by placing two to three females with a proven breeder of the same nutritional group and generation. Vaginal smears were taken daily during the breeding period. When sperm were noted in the vaginal smear, the subject was considered pregnant (day 1 of gestation) and was placed in an individual polycarbonate cage $(420 \times 200 \times 200 \mathrm{~mm})$ on day 19 of gestation. A $12.5 \mathrm{~mm}$ layer of Absorb-Dri bedding was placed in the cage at this time and left undisturbed for the entire litter period. At parturition, the numbers of live and still births were recorded. All pups were weighed within $24 \mathrm{~h}$ of birth.

Half the females were not mated, but were weighed daily to determine normal weight gain during the experimental period. Pregnant and non-pregnant animals were weighed to the nearest $1 \mathrm{~g}$ using a Sauter K1200 balance.

\section{Statistical analyses}

Weight gain for each $7 \mathrm{~d}$ period of pregnancy was compared by a two-way analysis of variance (ANOVA; nutritional group $\times$ days of pregnancy), using a repeated measure design. In addition, one-way ANOVA was carried out for the following measures: duration of pregnancy, initial weight at onset of pregnancy, weight gain and percentage of weight gain for the total gestational period, number of pups per litter and mean weights of pups. Scheffé tests were performed whenever ANOVA showed a significant difference.

RESULTS

\section{Duration of pregnancy}

Rehabilitated $\left(R_{1} / L P\right)$ animals had the longest gestation $(24.4 \pm 1 \cdot 7 \mathrm{~d})$ of the four groups; mean ( \pm SE) lengths of gestation (d) for the other groups were: $F_{n} / L P 23.2 \pm 0.7 d, F_{1} / L P$ $23.0 \pm 0.0 \mathrm{~d}$ and $\mathrm{F}_{\mathrm{n}} / \mathrm{C} 23.2 \pm 0.5 \mathrm{~d}$. ANOVA revealed a significant effect of group ( $F 5.66$, df 3,$53 ; P<0.01$ ). Scheffé simultaneous confidence intervals showed that $R_{1} / L P$ females had a significantly longer gestational period than $F_{n} / L P, F_{1} / L P$ or $F_{n} / C$ females $(P<0.05)$. No significant differences were found between the latter three groups. 


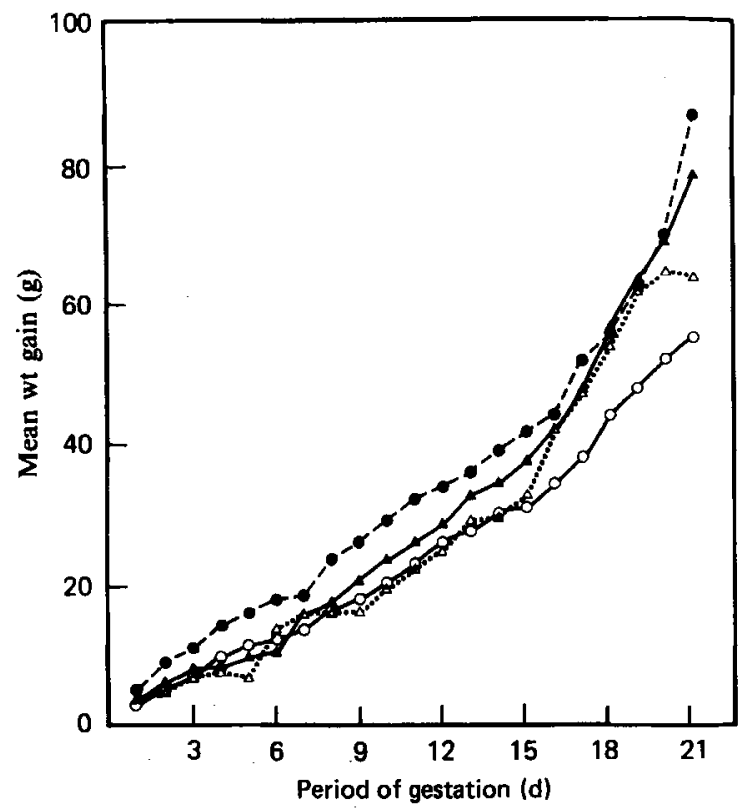

Fig. 1. Weight gains $(g)$ of animals during gestation $\left(O^{-O}\right) F_{n} / L P,\left(\Delta^{-}--\Delta\right) F_{1} / L P,\left(0^{--O}\right)$ $R_{1} / L P,(\Delta-\Delta) F_{n} / C$.

\section{Weight gain during pregnancy}

ANOVA of groups of non-pregnant animals did not demonstrate any significant differences in percentage of weight gain (weight gain divided by initial weight) during the $21 \mathrm{~d}$ of this experiment. Therefore, we report only results from pregnant subjects. Weights at onset of pregnancy were lower in malnourished animals than in well-nourished animals. Mean $( \pm \mathrm{SE})$ weights $(g)$ for well-nourished animals were: $R_{1} / L P 233 \cdot 1 \pm 15$ and $F_{n} / C 228.9 \pm 28.1$ and for the malnourished animals corresponding values were: $F_{1} / L P 194 \cdot 1 \pm 14$ and $F_{n} / L P$ $181 \cdot 3 \pm 23$. ANOVA showed that nutritional treatment had a highly significant effect on mean weight at the onset of pregnancy $(F 18 \cdot 3, \mathrm{df} 3,53 ; P<0 \cdot 001)$. Scheffé tests confirmed that the two well-fed groups each weighed significantly more than either of the two malnourished groups $(P<0.05)$ whereas there was no difference in weight at the onset of pregnancy between the two well-fed groups or between the two malnourished groups.

Animals given an adequate diet during pregnancy gained substantially more weight during the entire period of gestation than did malnourished animals (Fig. 1). ANOVA confirmed a significant effect of nutritional group in the two well-nourished groups $(F 6.08$, df 3,$53 ; P<0.01$ ). Scheffé tests showed that this effect was due to large differences between both the $\mathbf{R}_{1} / \mathrm{LP}$ and control $\left(\mathrm{F}_{\mathrm{n}} / \mathrm{C}\right)$ groups compared to the $\mathrm{F}_{\mathrm{n}} / \mathrm{LP}$ group $(P<0.05)$. Thus, in addition to weighing more at the onset of pregnancy, well-nourished females $\left(F_{n} / C\right.$ and $R_{1} / L P$ ) gained more weight during pregnancy than did malnourished females.

The results of ANOVA on weight gain during three individual periods of pregnancy are shown in Table 1 . During the first period (days $1-7)$, rehabilitated females $\left(R_{1} / L P\right)$ gained more weight than the other three groups. This difference in weight gain was greatest on day 7. Thus, there was a significant interaction between dietary group and time. During the second period (days 8-15), a significant effect of diet was also observed as a result of the continued advantage in weight gain among the $R_{1} / L P$ (rehabilitated) females. The well-nourished females $\left(F_{n} / C\right)$ gained weight more rapidly during this period than did the 
Table 1. Weight gain during pregnancy (analysis of variance)

\begin{tabular}{lccc}
\hline Period of pregnancy (d) & $1-7$ & $8-14$ & $15-21$ \\
\hline Diet & $F 2.362$ & $F 3.914^{*}$ & $F 2.911^{*}$ \\
& df 3,53 & df 3,53 & df 3,40 \\
Time & $F 73.035^{* *}$ & $F 131 \cdot 647^{* *}$ & $F 121 \cdot 831^{* *}$ \\
Diet $\times$ time & df 6,318 & df 6,318 & df 6,240 \\
& $F 1.674^{*}$ & $F 0.688$ & $F 3.055^{* *}$ \\
& df 18,318 & df 18,318 & df 18,240 \\
\hline
\end{tabular}

* $P<0.05, \quad$ ** $P<0.001$.

two groups of malnourished females $\left(F_{1} / L P\right.$ and $\left.F_{n} / L P\right)$. During the third period (days 16-22), the least weight gain occurred among the females with intergenerational malnutrition $\left(F_{n} / L P\right)$. The females with short-term malnutrition $\left(F_{1} / L P\right)$ gained rapidly during this period, 'catching up' to a normal range between days 16 and 20. Although this rapid growth spurt did not continue beyond day 20, the two groups receiving adequate protein $\operatorname{diets}\left(\mathrm{F}_{\mathrm{n}} / \mathrm{C}\right.$ and $R_{1} / L P$ ) grew most rapidly after day 20 . Thus, there was a significant interaction between stage of pregnancy and nutritional status during the third period of pregnancy.

\section{Pups}

The number of pups per litter was similar regardless of nutritional group. However, the percentage of still births among the offspring of females with postnatal malnutrition (16/44 or $36.4 \%)$ was significantly higher than in offspring of $F_{n} / C(4 / 259$ or $1 \cdot 7 \%), F_{n} / L P(6 / 146$ or $4 \cdot 1 \%)$ and $R_{1} / L P(4 / 74$ or $5.4 \%)$ females. $A 4 \times 2 \chi^{2}$ analysis showed a highly significant effect of group $\left(\chi^{2} 63.4\right.$, df $\left.4 ; P<0.001\right)$. When the $F_{n} / L P, F_{n} / C$ and $R_{1} / L P$ groups were pooled and compared to the $F_{1} / L P$ group in a $2 \times 2 \chi^{2}$ analysis, this difference was also highly significant $\left(\chi^{2} 62.3\right.$, df $\left.1 ; P<0.001\right)$. However, the mean weight of live pups was similar in all the groups.

\section{DISCUSSION}

This study demonstrated that rehabilitation after multigenerational malnutrition has a striking effect on pregnancy. $R_{1} / L P$ females had accelerated growth, and pregnancy was prolonged in those females who successfully gave birth. Females with continued malnutrition after several generations and those malnourished only postnatally did not show these phenomena. This finding implies that rehabilitation after birth may have adverse effects because of immaturity of metabolic control mechanisms, notably endocrine. For example, intra-uterine malnutrition is followed by subnormal thyroid function with reduced plasma levels of triiodo-thyronine (D. J. Friedman and F. J. Zeman, unpublished results). Persistence of this endocrine deficit after birth may account for the supra-normal weight gain observed in the rehabilitated rats (unpublished results), and the slower metabolic rate of thyroid insufficiency could also account for the rehabilitated females' longer pregnancies.

In contrast, females malnourished postnatally showed no reproductive deficiency, but had more still-born pups than did rats with intergenerational malnutrition. Thus, one result of long-term intergenerational malnutrition appears to be adaptation, which may include hormonal changes appropriate to increase survival under conditions of impaired nutrition. However, these long-term adaptive changes may prove disadvantageous when the females are rehabilitated on a $250 \mathrm{~g}$ protein $/ \mathrm{kg}$ diet. These preliminary findings suggest that rehabilitation in populations with chronic exposure to malnutrition should be carried out cautiously. 
Our study also emphasizes that weight gain per se during pregnancy is an inadequate measure of reproductive competence under a variety of nutritional conditions. Specifically, weight gain was accelerated in the rehabilitated group, yet reproductive performance was impaired. Similarly, the postnatally-malnourished females showed better weight gain than the intergenerationally-malnourished females, although the number of still births increased.

J. R. Galler was supported by The Medical Foundation, Boston, MA, and by Biomedical Research Support Grants NIH RR05380-18 and NIH RR05487-15. The authors thank Kathleen Propert for assistance in preparing this paper.

\section{REFERENCES}

Baird, D. (1947). Lancet 253, 531.

Baird, D. \& Illsley, R. (1953). Proc. R. Soc. Med. 46, 53.

Bernard, R. M. (1952). Edinburgh med. J. 59, 1.

Galler, J. R., Fox, J., Murphy, J. C. \& Melanson, D. E. (1979). Br. J. Nutr. 41, 611.

Stewart, R. J. C. (1972). P.A.H.O. Sci. Publ. 251, 33.

Stewart, R. J. C., Preece, R. F. \& Sheppard, H. G. (1973). Proc. Nutr. Soc. 32, 103A. 